\title{
On a PP/DP asymmetry in extraction
}

\author{
Valentina Bianchi \& Cristiano Chesi
}

\begin{abstract}
Preverbal subject DPs in English seem to allow for the extraction of a PP complement, but not of a DP complement stranding the preposition of. Assuming a top-down computation, we argue that an extracted PP cannot be re-merged within a criterial preverbal subject (in the sense of Rizzi 2006), but it can be re-merged within a non-criterial subject that has undergone full reconstruction. This 'delayed remerge' implies that in the preverbal position, the selectional requirement of the $\mathrm{N}$ head for a PP complement is not computed yet. This immediately accounts for the impossibility of P-stranding within a preverbal subject: the presence of the preposition implies that the computation of the N's selectional requirement has not been delayed.
\end{abstract}

\section{Introduction}

Chomsky $(1986,32)$ attributes to Adriana Belletti the observation that unexpected PP vs. DP asymmetries emerge in cases of apparent extraction from islands. In this paper we will be concerned with extraction from preverbal subjects, where stranding the preposition of - as in (1a) - yields a more deviant result than pied piping it - as in (1b): 
(1) a. * the man who [pictures of $t_{\mathrm{DP}}$ ] are on the table

b. he is the person of whom [pictures $t_{\mathrm{PP}}$ ] are on the table

(Chomsky 1986, (61a), (64))

This contrast is mysterious if preverbal subjects are absolute islands, as in the classic CED account (Huang 1982) and its recent minimalist reinterpretations (Takahashi 1994); as a matter of fact, this contrast has been either ignored or explained away as involving only apparent extraction (Longobardi 1991, Jurka 2010).

In this paper we will argue instead that (1b) is a real instance of whextraction, since it is sensitive to the nature of the subject. We will then show that the DP/PP asymmetry in extraction follows if we assume a topdown, left-to-right derivation (Chesi 2004/2012; Bianchi \& Chesi 2006, forthcoming), in which a moved phrase is first computed in the 'displaced' (non-thematic) position, it is subsequently stored in a memory buffer, and it is re-merged in the structure as soon as a selectional requirement is computed which triggers the projection of the corresponding thematic position. Specifically, the asymmetry will be traced to the possibility of delaying the completion of the subject DP up to the point where the subject is re-merged in its thematic position.

The paper is organized as follows: in $\S 2$ we critically review the 'no extraction' account of (1b), and we argue that it is inadequate, since it 
cannot explain the fact that sub-extraction is sensitive to the nature of the subject. In $\S 3$ we discuss the selectivity of subject island effects and we summarize the analysis proposed in Bianchi \& Chesi (2014) in terms of a top-down computation. In $\S 4$ we review Jurka’s (2010) experimental evidence on the DP/PP contrast, and we provide further experimental evidence from English. In $\S 5$ we argue that this contrast follows from the expectations introduced in the derivation by the selectional requirement of lexical heads: this insight can only be captured in a continuation-oriented, top-down computation, but not in a bottom-up approach. Finally, §6 provides a take-home summary and some concluding remarks.

\section{The 'no extraction' account}

Starting from Huang's (1982) Condition on Extraction Domains, the view has prevailed that preverbal subjects are strong islands for extraction, since they are in a non-selected position. In compliance with the CED and its subsequent reformulations (e.g. in Chomsky’s 1986 Barriers system), the unexpected (if marginal) possibility of extraction in (1b) has been explained away by reanalyzing it as a case of only apparent extraction.

In particular, Jurka (2010) argues that the pied piping case is not an instance of extraction: the PP is actually base-generated in a hanging topic position. Thus, the structure underlying example (2) is akin to (3): 
(2) [Of which cars] were [the hoods _ ] damaged by the explosion?

(Ross 1967:242, (4.253))

(3) *(Of) which cars was it the case that the hoods (of those cars) were damaged by the explosion? (Jurka 2010, 151, (10))

Under this account, the PP/DP asymmetry is explained by the fact that a left-peripheral DP, contrary to a PP, could not be interpreted as a hanging topic in this context.

A somewhat similar proposal had been advanced by Longobardi (1991, 8285) in order to account for cases of apparent extraction from unergative subjects in Italian. Longobardi noted that in (4), extraction from a definite subject is better than from an indefinite one:

(4)

$$
\begin{aligned}
& \text { quell'uomo politico, di cui ci ha telefonato *un segretario / } \\
& \text { ?il segretario } \\
& \text { that politician, of whom us has phoned a secretary / } \\
& \text { the secretary }
\end{aligned}
$$

Longobardi argued that when the subject is definite, there is no real whextraction: the definite DP, whose head is a relational noun, allows a 'possessive' interpretation in which the possessor remains implicit, as in (5): 
(5) A proposito di Maria, ci ha telefonato il segretario. speaking of Mary, us has phoned the secretary (= Mary’s secretary)

Hence, the variant of (4) with a definite subject can be rescued by interpreting the wh-phrase as a topic-like constituent, "with the article marginally acting as a resumptive position for it”, similarly to (5).

One problem with this assimilation is that 'possessor resumption' as in (5) is also possible with a partitive subject, cf. (6); on the contrary, a wh-PP cannot be related to a partitive subject, as shown in (7). This contrast is unexpected under Longobardi’s account.

(6) A proposito di Maria, ci ha telefonato uno dei figli.

speaking of Mary, us has phoned one of-the sons

(7) * Maria, di cui ci ha telefonato uno dei figli, ...

Mary, of whom us has phoned one of-the sons

The general problem with the 'no extraction' approach is that it does not predict any selectivity in the alleviation of subject island effects. On the contrary, it has been conclusively shown that the alleviation of island effects is selective and depends on the nature of the subject. In $\S 3$ we briefly review this evidence and we lay out our own approach to subject islands, so as to provide the background for our analysis of the PP/DP asymmetry. 


\section{A revised perspective on subject islands}

\subsection{The selectivity of subject island effects}

In the pre-minimalist phase, despite the overwhelming prevalence of a CED(-type) account, some counter-examples of licit extractions from subjects had occasionally been noted in the literature. Some scholars even argued that subject islands are an illusory constraint (e.g. Levine \& Sag 2003) or that they can be explained in terms of performance (e.g. Kluender \& Kutas 1993, Hofmeister \& Sag 2010). In the mainstream approach to subject islands, however, the turning point was constituted by Chomsky (2008) (first circulated in 2004), which argued that preverbal subjects can be extracted from when they are internal arguments (i.e., unaccusative or passive subjects), but not when they are external arguments:

(8) a. * Of which car did [the (driver, picture) $t$ ] cause a scandal?

(external argument)

b. Of which car was [the (driver, picture) $t$ ] awarded a prize?

(internal argument)

The subsequent minimalist literature has proposed different assessments of the data: we refer to Jurka (2010) and Bianchi \& Chesi (2014) for general 
reviews. Crucial for our argument is the observation that, if the acceptable examples involved only apparent extraction, no selectivity of any type would be expected: in (8), for instance, there is no clear reason why a hanging topic PP could be interpretively connected to an internal argument but not to an external argument. This observation leads us to conclude that real extraction is involved.

In Bianchi \& Chesi (2014), we argued that the possibility of extraction is sensitive to the categorical vs. non-categorical status of the subject, in the sense of Ladusaw (1994). Consider the following contrast:

(9) [Context: An art collector has ordered reproductions of a number of masterpieces: some big-size reproductions and a small-size one for each.]

a. Of which masterpiece is [one reproduction $t_{\mathrm{PP}}$ ] already available?

b. $\quad$ ?* Of which masterpiece is [one reproduction $t_{\mathrm{PP}}$ ] absolutely perfect ${ }^{1}$

\footnotetext{
${ }^{1}$ An anonymous reviewer pointed out a parallel contrast in case of PP extraposition:

(i) Just [one reproduction_] is available [pp of this masterpiece].

(ii) *Just [one reproduction_] is perfect [pp of this masterpiece].

Guéron (1980) first noticed that only the subject of a presentational clause (and not the presupposed subject of a predication, Guéron 1980:254) allows for PP extraposition. This is consistent with the analysis to be presented below.
} 
In (9a), the predicate is stage-level (denoting a non-permanent property of the subject) and the subject receives a non-presuppositional interpretation (i.e. one that does not presuppose the existence of a referent): following Ladusaw (1994), this corresponds to a thetic LF structure (10), in which the subject is reconstructed within the predicative nucleus of the clause, in the scope of existential closure.

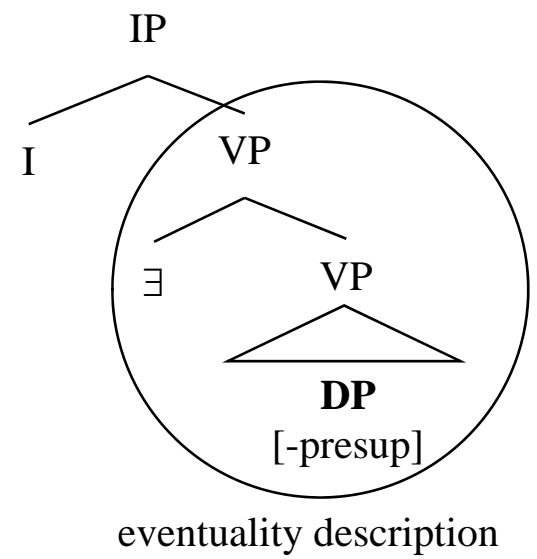

In (12a), instead, the predicate is individual-level (denoting a characterizing property of the subject): in this case, the subject cannot be reconstructed into its thematic position, ${ }^{2}$ but it must be fully completed and interpreted in its surface position, outside the predicative nucleus of the clause, giving rise to a categorical LF structure (11):

\footnotetext{
${ }^{2}$ This corresponds to Diesing's (1992) hypothesis that individual-level predicates are 'control' predicates, whose subject cannot occur in a VP-internal position.
} 


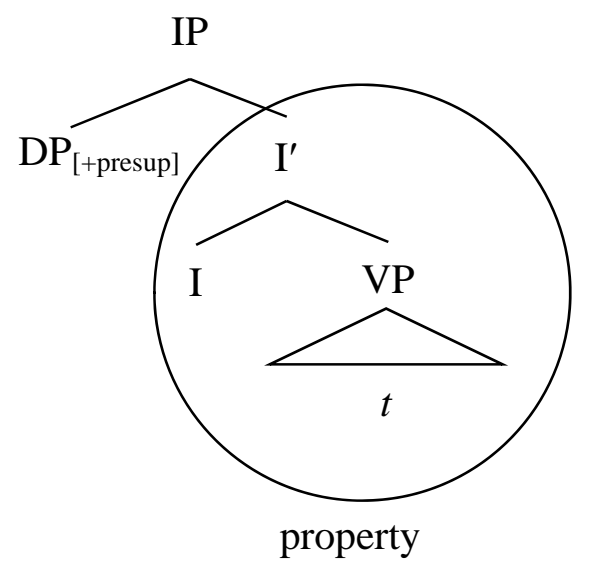

In Bianchi \& Chesi (2014), we argued that the categorical LF structure (11) is implemented by means of the Subject Criterion (in the sense of Rizzi 2006). The preverbal subject enters a Spec-head relation with a dedicated functional head, $\mathrm{Subj}^{0}$, which induces a "freezing” effect: the subject can neither move away nor be reconstructed from the criterial position Spec, SubjP. On the contrary, in a thetic structure like (10) the preverbal subject occupies a lower, non-criterial position (cf. Kiss 1996 and Cardinaletti 2004), and it undergoes reconstruction into the thematic position. The empirical contrast exemplified in (9) will be further supported by the experimental evidence discussed in $\S 4$.

The possibility of extracting from the non-criterial subject of a thetic structure, but not from the criterial subject of a categorical structure, is captured by the following Extraction from Subject Constraint: 


\section{(12) Extraction from Subject Constraint (ESC)}

Only a subject occupying a thematic position at the LF interface is transparent for extraction.

One immediate question raised by the ESC (12) is why extraction from a subject should be sensitive to its covert position at the syntax-semantics interface, rather than to its overt position. As a matter of fact, in a framework with separate overt and covert cycles, the ESC could not even be stated. In the next section we argue that the ESC is instead a natural constraint in a top-down computation.

\subsection{Subject islands from a top-down perspective}

As discussed in Bianchi \& Chesi (2014), the ESC is a very unnatural constraint in a bottom-up derivation, but it falls out naturally if we adopt instead a top-down, left-to-right oriented computation.

The framework that we will adopt is presented in detail in Chesi (2012); here we will informally summarize the essential points. We wish to stress that this is not a processing model, but a generation model that simply reverses the directionality of the structure building operations. Importantly, 
in this model the syntactic and semantic computation proceed in parallel and are divided in phases (to be defined below). Given the top-down orientation, this implies that a categorical subject is computed and interpreted in its criterial position, outside the predicative nucleus; as a consequence, the thematic position is filled by a bound variable. In thetic structures, instead, the completion and interpretation of the non-criterial subject can be delayed until the subject is re-merged in the thematic position: this is the top-down implementation of reconstruction. This basic difference in the derivation of the subject dependency will be shown to account for (a) the islandhood of categorical subjects and the transparency of non-categorical ones; (b) the impossibility of stranding a preposition under extraction (cf. (1a)).

In a top-down computation, lexical insertion (external merge) is triggered by categorial selection and must be satisfied locally, right after the selecting lexical item has been computed: this results in a "left-to-right" derivation. Every lexical item is endowed with features that can be selected, and can also be endowed with features that select. For instance, an unaccusative verb is minimally marked in the lexicon with a categorial feature "V" and a select feature “=DP” (e.g. [v=DP fall]). This implies that the assigned thematic role is licensed right after the verb has been computed.

An argumental DP or PP appearing in a non-thematic position - for instance, a preverbal subject - is licensed there by a functional feature. This, however, only licenses a subset of the features of the phrase: in particular, the features qualifying it as an argument are unselected (i.e. unexpected), 
and hence, the phrase is not licensed as an argument of any specific lexical head. For this reason, after being computed in its surface position, the phrase is stored in a memory buffer, and it is discharged and re-merged into the structure when a selecting lexical head has been computed that licenses its thematic position. Note that, contrary to the bottom-up derivation, the 'derived' (non-selected) position is computed before the thematic (selected) position.

In this system, phases are defined as follows:

\section{(13) Phase definition}

A phase as a subpart of the top-down computation in which all the functional and selectional features associated to a lexical head are computed. ${ }^{3}$ Each phase is endowed with a local memory-buffer.

A phase is completed and closed right after the last selectional feature of its head (if any) has been computed: this will introduce in the derivation the expectation for the selected constituent, whose expansion will constitute the next computational phase.

The phase local memory buffers implement the cyclicity of movement dependencies. Once a phase is completed (that is, all the selectional requirements of its lexical head have been projected), its memory buffer

\footnotetext{
${ }^{3}$ Intuitively, this corresponds to the computation of an extended projection in the sense of Grimshaw (1990). For instance, a 'CP phase' is a phase headed by a lexical V head. Here we will retain the standard labels CP and DP for simplicity.
} 
must be empty, or else its content must be transmitted to the memory buffer of the next phase. At the end of the derivation the memory buffer of the last phase must be empty, i.e. all dependencies must have been discharged. Islandhood is expressed by the distinction between phases that are computationally nested (islands) vs. sequential. A phase is nested whenever its computation interrupts the computation of the superordinate phase: this is exactly the case of a preverbal subject, which interrupts the computation of the containing clausal phase. On the other hand, a phase is sequential when it is the last complement selected by the head of the superordinate phase, i.e., it corresponds to the right recursive branch of the tree.

Crucially, the transfer of the content of a memory buffer from phase to phase is sensitive to the nested vs. sequential distinction, in virtue of the following Inheritance Constraint:

(14) Inheritance constraint (Chesi 2012; Bianchi \& Chesi 2006, 2014)

a. When a phase head is computed, its last selectional feature triggers the projection of a sequential phase: the first phase is closed and the projected phase is computed sequentially. The sequential phase inherits the memory buffer of the preceding phase.

b. A nested phase, instead, is a phase that constitutes the expansion of a functional feature (or of a selectional feature which is not the last one): it is computed while the superordinate phase is still not 
concluded, because its lexical head has not been (fully) computed. A nested phase cannot inherit the memory buffer of the superordinate phase.

The conceptual motivation for the ban against inheritance for nested phases (14b) comes from considerations of computational complexity. Briefly, from an algorithmic perspective, a movement dependency significantly increases the complexity of the problem of which dominance relations have to be associated to a given set of precedence relations: at worse, any item could be remerged into any lower position. Hence, the complexity order of the problem is exponential with respect to the number of nodes to be recursively expanded. This is not a computationally exploitable solution, since the growing rate of the searching algorithm would make the problem quickly intractable (see Chesi 2012, 159-170 for thorough discussion). By restricting inheritance to phases that are computed sequentially, the exponential increase of complexity is avoided.

Consider now the illegal extraction in (9b), repeated here as (15):

?* Of which masterpiece is [one reproduction $t_{\mathrm{PP}}$ ] absolutely perfect?

The computation would consist of the following derivational steps, which are schematically illustrated in (16): 
(16)

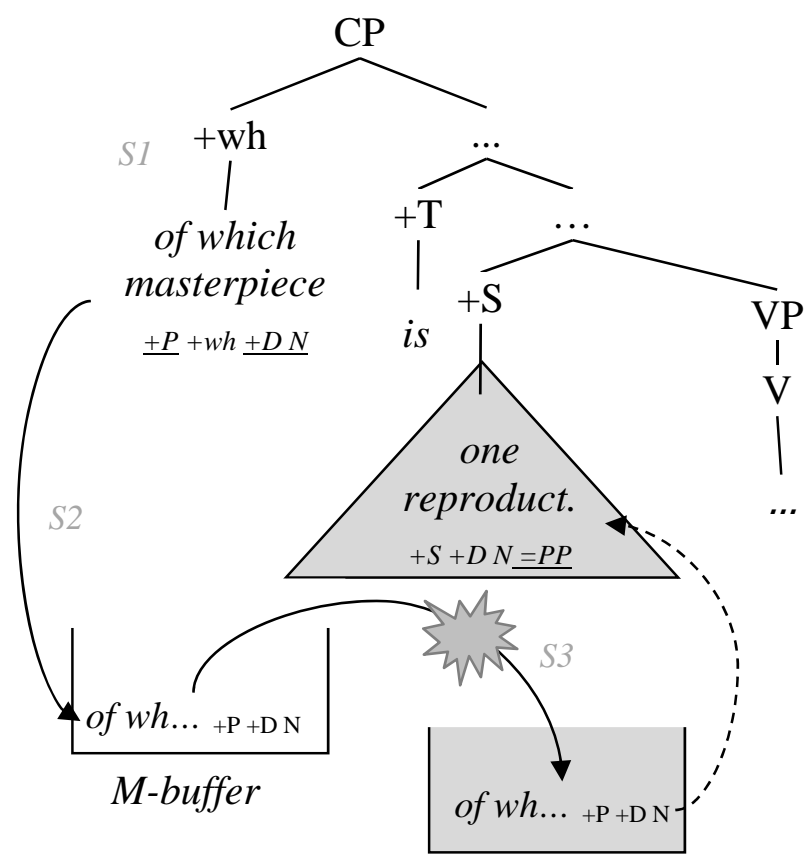

S1. The system initializes a CP phase.

S2. The wh-PP is computed in a left-peripheral position (spec,CP); since in this position it is not licensed by any selectional requirement of a lexical head, it is stored in the memory buffer associated with the CP phase. ${ }^{4}$

S3. The subject DP phase is computed: its head (reproduction) introduces a selectional requirement for a $\mathrm{PP}^{5}$ (the active features in $\mathrm{S} 3$ are underlined: $=\mathrm{PP}$ is a selecting feature). However, for the wh-PP to be remerged within the subject DP phase, as the complement of

\footnotetext{
${ }^{4}$ The active features in S2 are underlined: these features trigger movement, since they are unselected - hence unlicensed - in the initial position.

${ }^{5}$ Notice that having or not a selectional requirement on a $\mathrm{N}$ head is not an option in generation. It is true, as noticed by an anonymous reviewer, that such selectional requirement is generally considered optional, but this simply means that our lexicon is ambiguous at the select feature level: from our perspective "reproduction" comes in two favour in the English lexicon: [N=Pp reproduction] and [N reproduction]. In this derivation, the first item is picked up from the lexicon. This removes any ambiguity or optionality during the rest of the derivation.
} 
reproduction, the DP phase should inherit the memory buffer of the matrix CP phase. Crucially, this step is disallowed by the inheritance constraint (14b). Therefore, the wh-PP cannot be discharged, and the derivation fails to converge.

The inheritance constraint (14b) seems to predict that preverbal subjects are absolute islands. Indeed, when the predicate is individual-level, as in (15), no alternative derivation is possible, because the criterial subject must be fully completed and interpreted in its surface position (only a bound variable is re-merged in the thematic position).

Consider now extraction from a non-criterial subject, as in (9b), repeated here as (17):

(17) Of which masterpiece is [one reproduction $t_{\mathrm{PP}}$ ] already available?

In this case, an alternative derivation is possible: as the predicate is stagelevel, the subject can be reconstructed within the predicative nucleus of the clause, giving rise to a thetic structure. In the top-down derivation, reconstruction consists in fully re-merging the subject DP in its thematic position and interpreting it there. It is then possible to avoid the problematic step S3 of (16) by delaying the re-merge of the wh-PP inside the subject DP 
until after the subject DP itself has been re-merged in the selected thematic position. This is allowed by the following Delay Principle: ${ }^{6}$

(18) Delay Principle

Discharge of a dependency into a moved constituent $\alpha$ can be delayed until $\alpha$ itself has been discharged from the M-buffer.

The derivation of (17) will then proceed through the following steps (again, we refer to Bianchi \& Chesi 2014 for a detailed presentation):

(19)

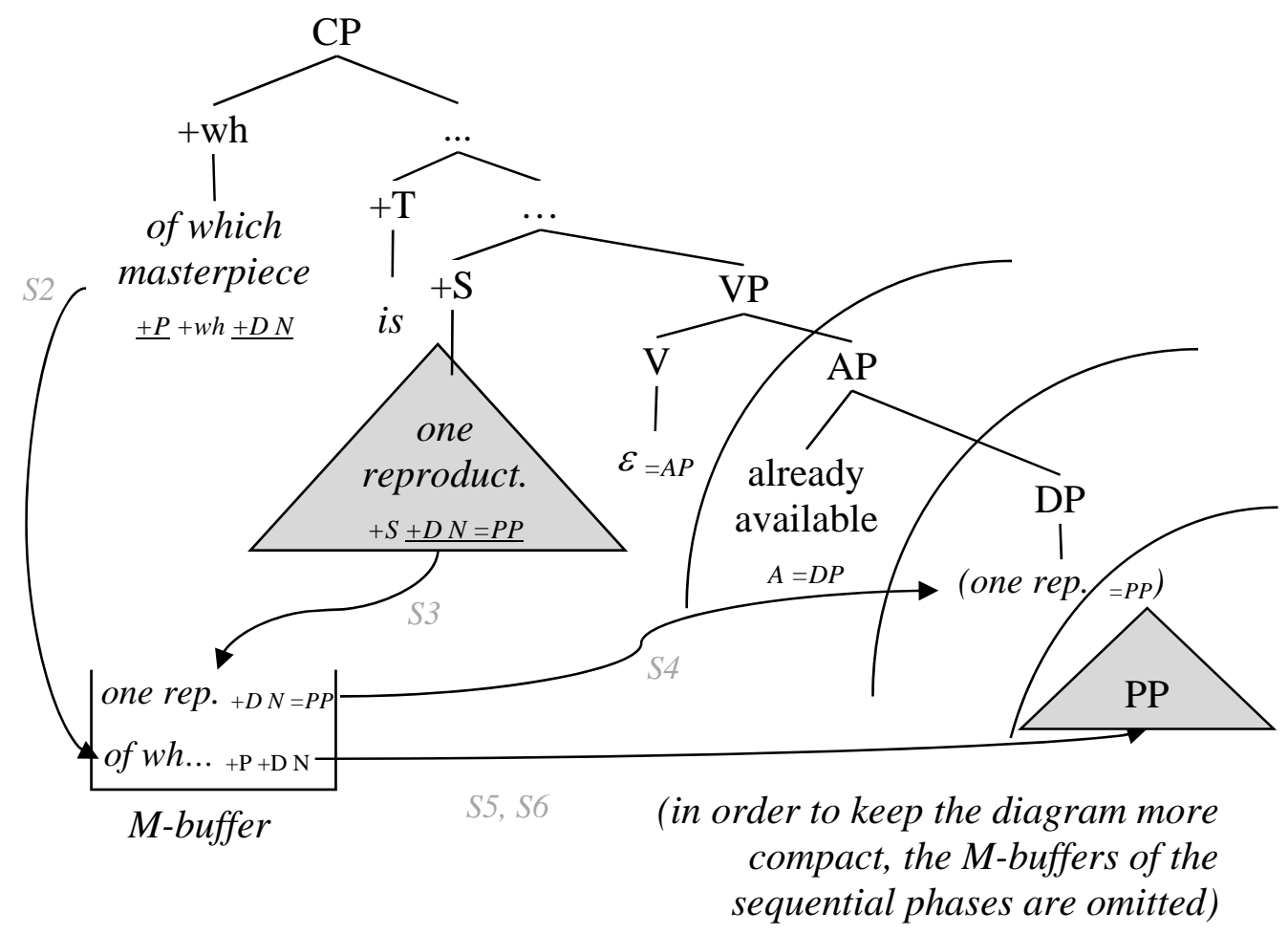

${ }^{6}$ The Delay Principle (18) is independently motivated by the existence of remnant movement; see Bianchi \& Chesi (2014) for discussion. 
S1. The system initializes a CP phase.

S2. The wh-PP is computed in a left-peripheral position (spec,CP). Since in this position it is not licensed by any selectional requirement of lexical head, it is stored in the memory buffer associated with the CP phase.

S2. The subject DP phase is computed. Its head (reproduction) introduces a selectional requirement for a PP.

S3. The completion of the subject DP phase is delayed (by (18)), and the incomplete DP is stored in the memory buffer.

S4. The adjective head available is computed, and it selects a DP: this selectional requirement triggers the re-merge of the subject DP in the thematic position.

S5. The re-merged DP phase is a sequential phase: hence, by (14a) it can inherit the memory buffer of the matrix CP phase, containing the stored wh-PP.

S6. The wh-PP is discharged within the DP, satisfying the selectional requirement of the noun head reproduction (delayed completion).

As a result, at the end of the derivation all the dependencies have been discharged, and the derivation converges.

In sum, the selective possibility of extraction rests on the ultimately interpretive requirement for a thetic interpretation of the clause: this accounts for the well-known variability and unstableness of grammaticality 
judgments (cf. Jurka 2010; see $\S 4.3$ for more discussion). Despite its apparent 'interface' status, the ESC is reduced to the interaction of two independently motivated derivational principles: the inheritance constraint (14) and the delay principle (18). This is allowed by the fact that the syntactic and semantic top-down computations proceed in parallel and are crucially divided in phases.

Building on this analysis of subject islands, we will now turn to consider the DP/PP asymmetry illustrated in (1) above.

\section{The PP/DP asymmetry: experimental evidence}

Jurka (2010) offers interesting experimental evidence that compares piedpiping vs. preposition stranding in extraction from both objects and subjects. The experimental paradigm is reported in (20) (Jurka 2010:153, (13)):

(20) a. subject, no extraction Phil wondered whether [a documentary about healthcare] had swayed the voters last year.

b. subject, pied-piping

Phil wondered [about which topic] [a documentary _] had swayed the voters last year. 
c. subject, no pied-piping

Phil wondered [which politician] [a documentary about_]

had swayed the voters last year.

d. object, no extraction

Phil wondered whether Scott had filmed [a documentary

about healthcare] last year.

e. $\quad$ object, no pied-piping

Phil wondered [which topic] Scott had filmed [a documentary about_] last year.

f. $\quad$ object, pied-piping

Phil wondered [about which topic] Scott had filmed [a

documentary _] last year.

The experimental task was a grammaticality judgement on a 7-point Likert scale. Despite great variability among participants, a general pattern clearly emerged, showing that pied-piping decreases acceptability in extraction from objects, but increases it in the case of extraction from subjects: ${ }^{7}$

\section{(21) Descriptive mean results}

(scale values: 1 bad - 7 good; Jurka 2011, 154)

\footnotetext{
${ }^{7}$ Jurka points out that "The prescriptive rule some speakers might remember as prohibiting ending sentences with a preposition would bias against this [i.e. the stranding] condition.” (Jurka 2010,154). Notice that this would only bias them against stranding a preposition within an object, not within a subject, which is not clause-final. The experimental results, however, do not comply with this prescriptive rule.
} 


\begin{tabular}{llll}
\hline & no extraction & pied-piping & P-stranding \\
\hline Subject & 6.24 & 3.29 & 2.51 \\
Object & 6.54 & 3.86 & 5.08 \\
\hline
\end{tabular}

Jurka found a highly significant Subject vs. Object x Extraction interaction when the preposition is stranded, while in the pied-piping condition, the interaction is not significant (Sub/Obj*Extraction in the pied-piping condition: $\mathrm{F}=24.849 \quad \eta^{2}=.184 ; \quad$ Sub/Obj*Extraction in the P-stranding condition: $\left.\mathrm{F}=302.715 \eta^{2}=.733\right)$.

He therefore concludes that P-stranding leads to perceived ungrammaticality for most speakers in the case of extraction from subjects:

$(22)^{\text {?* }}$ [Which cars] were [the hoods of _ ] damaged by the explosion?

(Jurka 2010, 151)

Note however that, because of Jurka's starting hypothesis, the experimental materials did not factor out the distinction between stage-level and individual-level predicates which, as discussed in §3, actually influences the islandhood of preverbal subjects. For this reason, we designed a new experiment in which both the nature of the preverbal subject and the DP/PP 
contrast are taken as potentially relevant factors affecting the acceptability of extraction.

\subsection{Experimental set-up}

The experimental paradigms implemented a $2 \mathrm{X} 2$ design, where Pstranding and pied-piping were compared in cases of wh-extraction from a preverbal subject, both with individual-level and with stage-level predicates. The two types of predicates were discriminated by the possibility of cooccurrence with phasal adverbs like 'already' and 'still'; we also provided a small context in order to facilitate the expected reading (thetic with stagelevel predicates, categorical with individual-level ones). Intransitive, adjectival and passive predicates were tested. The experimental paradigms are exemplified in (23):

(23) Context: An art collector has ordered reproductions of a number of masterpieces: some big-size reproductions and a small-size one for each

\section{a. [i-level, pied-piping]}

Of which masterpiece is [one reproduction _ ] absolutely perfect?

b. [i-level, P-stranding]

Which masterpiece is [one reproduction of _ ] absolutely perfect?

\section{c. [s-level, pied-piping]}


Of which masterpiece is [one reproduction _ ] already available?

\section{d. [s-level, P-stranding]}

Which masterpiece is [one reproduction of _ ] already available?

Our expectations were the following:

(a) with individual-level predicates, extraction should be unacceptable independently of stranding vs. pied-piping the preposition;

(b) with stage-level predicates, extraction should be sensitive to P-stranding vs. pied-piping, favoring the latter option.

The data were collected with a controlled judgment elicitation technique.

The experimental items consisted of 12 paradigms like (23), with 4 variable combinations each (2 preposition positions X 2 predicate types). The items were divided into four different experiments (Latin Square design): in every experiment each of the four conditions (a-d) was tested with 3 items, so that only one example was extracted from each paradigm. The items were interspersed with a number of fillers (of various degrees of acceptability) more than three times bigger than the number of the items; items and fillers were presented in a randomized order.

The subjects were presented the data via an on-line interface implemented with Osucre (Van Acker 2007). They were asked to indicate the degree of acceptability of each presented sentence on a continuous bar with 400 points. Figure 1 shows a snapshot of the data presentation: 
Context: An art collector has ordered reproductions of a number of masterpieces: some big-size reproductions and a small-size one for each. of which masterpiece is one reproduction absolutely perfect?

horrible $\longrightarrow$

Next page

Figure 1 Experiment screenshot

The experimental subjects were 23 adult native speakers from UK, who were recruited personally or by e-mail by the investigators. None of them performed all the four experiments. The total number of concluded tests was 32, yielding a total of 384 acceptability judgments.

\subsection{Results}

The results were analyzed with $\mathrm{R}$ using a within-subject analysis, 2-way ANOVA.

First of all, we observed a great variability in grammaticality judgments (Figure 2): 


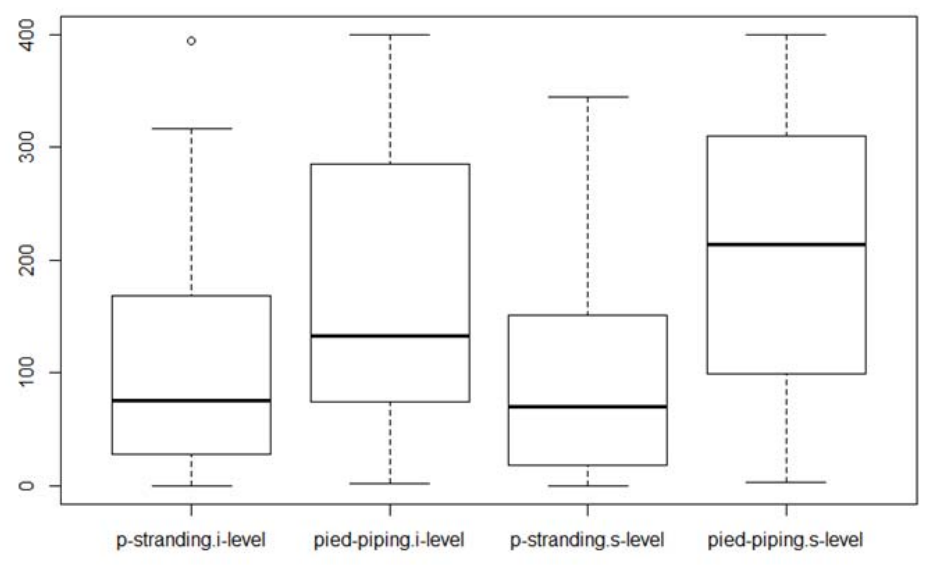

Figure 2 Box Plot

Despite this great variability, two main effects emerged clearly: first, there is a significant effect on pied-piping conditions vs. P-stranding conditions $(F(1,22)=22.204 p<0.01)$, with the latter judged significantly worse than the former. Second, although globally there is no significant dependence of acceptability on the predicate type $(\mathrm{F}(1,22)=4.200 p=0.053)$, we found a significant effect on the interaction between pied-piping vs. P-stranding and predicate type $(\mathrm{F}(1,22)=4.997 p=0.036)$, cf. also Figure 3 . 


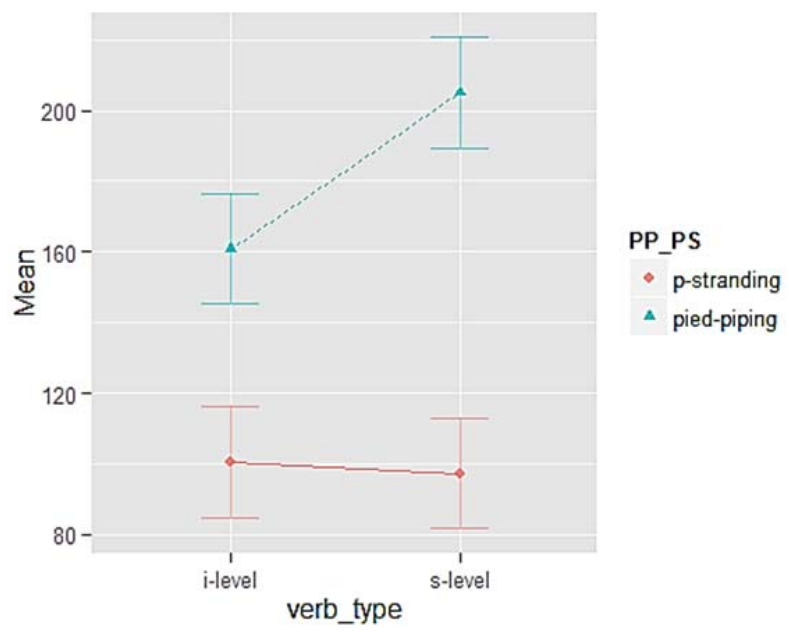

Figure 3 Variables interaction

The amelioration due to pied-piping is boosted by the significant increase of acceptability when pied-piping applies in items with stage-level predicates (as the $t$ test on s-level items shows: $\mathrm{t}=-7.829$, $\mathrm{df}=161.6, \mathrm{p}$-value $=$ 6.127e-13, as opposed to the weaker significance of the same pied-piping vs. P-stranding contrast on i-level items: $\mathrm{t}=-4.314$, $\mathrm{df}=168.6$, $\mathrm{p}$-value $=$ 2.719e-05).

\subsection{Discussion}

As discussed in Bianchi \& Chesi (2014), the great variability of the obtained acceptability judgements is to some extent inevitable. First, despite our effort in inducing a stage-level or an individual-level interpretation of the given predicates, some degree of ambiguity is ineliminable (cf. Diesing 
1992). Second, with stage-level predicate both a presuppositional (categorical) and a non-presuppositional (thetic) interpretation are possible: this further ambiguity too may not have been entirely eliminated by the facilitating contexts that we provided for each item. Third, we must also consider the fact that the experimental items were not in our own native language and, even though the items have been checked by a native speaker of English, they may have involved some subtle deviations from full naturalness. This said, the main effects we found imply that

(a) concerning the effect of subject type, our prediction is borne out: if we compare only the more acceptable pied piping conditions (second and fourth boxes from the left in Figure 2), subjects of stage-level predicates are much more transparent for extraction than the categorical subjects of individual-level predicates (as the Paired t-test on pied-piped items shows: $\mathrm{t}=-2.8089, \mathrm{df}=22$, $\mathrm{p}$-value $=0.01$;

(b) our results also confirm the relevance of the pied-piping vs. P-stranding opposition: in particular, if we compare the pied-piping vs. stranding conditions with stage-level predicates (third and fourth boxes from the left in Figure 2), the effect is strongly significant (Paired t test on grammaticality grouped by subjects: $\mathrm{t}=-4.8132, \mathrm{df}=22, \mathrm{p}$-value $=$ 9.328e-05). ${ }^{8}$

\footnotetext{
${ }^{8}$ An anonymous reviewer was concerned with the possibility that the results might have been affected by prescriptive effects. As discussed in note 7, in Jurka’s study P-stranding is more acceptable in object position, despite normative grammar; in subject position, the
} 
Thus, both of our expectations were borne out.

\section{Explaining the DP/PP asymmetry}

It is easy to show that the DP/PP asymmetry already illustrated in (1) (featuring a stage-level predicate), and confirmed by our experimental results, falls out immediately from the top-down analysis summarized in §3. Consider again the basic DP/PP contrast that emerged in the stage-level conditions of our experiment, repeated here as (24):

(24) a. Of which masterpiece is [one reproduction _ ] already available?

b. ?* Which masterpiece is [one reproduction of _ ] already available?

Recall from $\S 3$ that a wh-phrase is first computed in its displaced position and is later re-merged in a thematic position when the selecting lexical head has been processed. Given the hypothesis that the preverbal subject is an “impenetrable” domain (by (14b)), the possibility of (24a) was explained along the following lines:

(i) the subject is left incomplete in the preverbal position, with the selectional requirement for a PP unsaturated;

asymmetry is exactly the reverse. This suggest that the prescriptive effects do not significantly affect the subjects’ grammaticality judgements. 
(ii) the subject then undergoes full reconstruction into a thematic position;

(iii) at that point, the noun's selectional requirement for a PP is processed and the wh-PP is re-merged (delayed completion).

The hypothesis of delayed completion immediately accounts for the impossibility of stranding the preposition within the preverbal subject, as in (24b). Crucially, the presence of the preposition implies that the noun's selectional requirement for a PP has already been processed within the preverbal subject phase, i.e. it has not been delayed. ${ }^{9}$ Thus, the impossibility of P-stranding virtually falls out as a corollary of the proposed analysis.

\section{Summary and further prospects}

This paper has been concerned with a tiny and apparently negligible empirical fact: certain subject islands allow for extraction of a PP, but not for the extraction of a DP stranding a preposition. Despite its subtlety, this contrast is quite robust, as shown by the results of Jurka's (2010) experiment and of our own experiment (§4): this suggests that a real grammatical constraint is involved. Unfortunately, the standard bottom-up

\footnotetext{
${ }^{9}$ Note that, even if in (24b) the preposition selected for the wh-DP, the latter cannot be remerged as a complement of the preposition of, because the preverbal subject constitutes a computationally nested phase, which cannot inherit the memory buffer of the matrix CP phase (by (14b)).
} 
view of the syntactic derivation gives us no hint of what the relevant constraint could be.

We have argued that the pied piping / stranding contrast follows naturally from a top-down derivation of extraction from subjects, which at the same time also accounts for the selectivity of subject island effects. The main points of the analysis can be summarized as follows:

(i) Preverbal subjects are absolute islands: this is because they constitute computationally nested phases, and as such, they cannot inherit a whdependency from the containing (matrix) phase.

(ii) The acceptability of extraction is contingent upon the possibility of delaying the completion of the subject DP until after the latter has been re-merged in the thematic position: the phase in the thematic position can inherit the wh-dependency of the matrix phase and hence allows for re-merge of the extracted $w h$-PP.

(iii) P-stranding within the preverbal subject DP is incompatible with delayed completion: this accounts for the robust prohibition against Pstranding within a preverbal subject.

(iv) Delayed completion, however, is only allowed when the subject receives a thetic interpretation, i.e. it is the non-presuppositional subject of a stage-level predicate, which is totally reconstructed and is ultimately interpreted within the predicative nucleus of the clause (Bianchi \& Chesi 2014). 
Our next step will be to examine Adriana Belletti’s original contrast w.r.t. adjunct islands, where she noted the opposite PP/DP asymmetry: here, a DP is better extracted than a PP, i.e. (25a) is more acceptable than (25b).

(25) a. Who did they leave [before speaking to $t_{\mathrm{DP}}$ ]?

b. To whom did they leave [before speaking $t_{\mathrm{PP}}$ ]?

(Chomsky 1986, (62))

This contrast too has received a 'no movement' account. Cinque (1990, Ch. 3) analysed (25a) as involving a representational $A^{\prime}$-chain with a null resumptive pronoun instead of a trace: by hypothesis, a representational chain is not sensitive to islands. The unacceptability of (25b) as compared to (25a) was explained by the hypothesis that null resumptive pronouns can only be of category DP. Interestingly, however, Truswell (2007) has shown that adjunct island effects too are selective (again, contrary to the predictions of the no movement account): an adjunct clause can be transparent for extraction if the event it denotes is identified with an event position in the matrix predicate. This interpretive constraint is reminiscent of the thetic/categorical divide which conditions subject island effects, in that the transparent subject of a thetic structures is also interpreted as part of 
the event description. We leave this intriguing parallelism for future research.

\section{References}

Bianchi ,Valentina \& Cristiano Chesi. 2014. Subject islands, reconstruction, and the flow of the computation. To appear in Linguistic Inquiry Vol. 45.4 (Fall of 2014)

Bianchi, Valentina \& Cristiano Chesi. 2006. Phases, left branch islands, and computational nesting. UPENN Working Papers in Linguistics 12.1, $15-28$.

Chesi, Cristiano. 2012. Competence and Computation: toward a processing friendly minimalist Grammar. Padova, Unipress. (Revised version of 2004 doctoral dissertation).

Chomsky, Noam. 1986. Barriers. Cambridge, MA: The MIT Press.

Chomsky, Noam. 2008. On phases. In Robert. Freidin, Carlos. P. Otero \& Maria Luisa Zubizarreta (eds.), Foundational Issues in Linguistic Theory,133-166. Cambridge, MA: The MIT Press.

\footnotetext{
* We are very grateful to, Elisa di Domenico, Cornelia Hamann and Simona Matteini for having invited us to contribute this paper to honour Adriana Belletti's 60th birthday. We wish to thank Alison Duguid for checking our experimental sentences, Nicoletta Biondo and Luca Cilibrasi for help in finding experimental subjects. For much discussion and insightful comments, we are indebted to Ciro Greco, Luigi Rizzi, Ur Shlonsky. This being a celebration paper for her, Adriana was not aware of its preparation, but she has inspired much of it, as well as much of our previous work, and will no doubt keep inspiring us in the future.
} 
Cinque, Guglielmo. 1990. Types of A'-dependencies. Cambridge, MA: The MIT Press.

Diesing, Molly. 1992. Indefinites. Cambridge, MA: The MIT Press.

Grimshaw, J. 1990. Extended projection. Ms., Brandeis University.

Guéron, Jacqueline. 1980. On the syntax and semantics of PP extraposition. Linguistic Inquiry, 11(4): 637-678.

Hofmeister, Philip \& Ivan A. Sag. 2010. Cognitive constraints and island effects. Language 86, 366-415.

Huang C. T. James. 1982. Logical Relations in Chinese and the Theory of Grammar. Cambridge, MA: Massachusetts Institute of Technology dissertation.

Jurka, Johannes. 2010. The importance of being a complement. Ph.D thesis, University of Maryland.

Kiss, Katalin É. 1996. Two subject positions in English. The Linguistic Review 13, 119-142.

Kluender, Robert \& Marta Kutas. 1993. Subjacency as a processing phenomenon. Language and Cognitive Processes 8, 573-633.

Ladusaw, William. 1994. Thetic and categorical, stage and individual, weak and strong. Proceedings of SALT 4.

Levine, Robert, \& Ivan Sag. 2003. Some empirical issues in the grammar of extraction. In Proceedings of the 10th International Conference on Head-Driven Phrase Structure Grammar, ed. by Stefan Müller, 236256. Stanford, CA: CSLI. 
Longobardi, Giuseppe. 1991. Extraction from NP and head government. In: Alessandra Giorgi \& Giuseppe Longobardi, The Syntax of Noun Phrases, Cambridge University Press, 57-112.

Moving on, 97-133. Cambridge, MA: MIT Press.

Pollard, Carl \& Ivan Sag. 1994. Head-driven Phrase Structure Grammar. Chicago \& London: The University of Chicago Press.

Rizzi, Luigi. 2006. On the Form of Chains: Criterial Positions and ECP Effects. In Wh-movement:

Ross, John Robert, 1967. Constraints on Variables in Syntax. Cambridge, MA: Massachusetts Institute of Technology dissertation.

Stepanov, Arthur. 2001. Cyclic domains in syntactic theory. Ph.D. dissertation, University of Connecticut, Storrs.

Stepanov, Arthur. 2007. The end of CED? Minimalism and extraction domains. Syntax 10:80-126.

Takahashi, Daiko. 1994. Minimality of movement. Ph.D. dissertation, University of Connecticut.

Truswell, Robert. 2007. Extraction from adjuncts and the structure of events. Lingua 117, 1355-1377. 